\title{
Efficient Multi-path Routing Protocols in Wireless Sensor Networks
}

\author{
Si-Gwan Kim
}

\begin{abstract}
Small-sized, cheap, and power-limited sensors that possess sensing, signal processing and wireless communication capabilities is becoming popular for the wireless sensor networks. Due to the limited resources and energy constraints, efficient routing algorithms should be employed in sensor networks. In this paper, we propose a node-disjoint multi-path hexagon-based routing algorithm. We divide the given messages into 4 segments, which are routed to 4 node-disjoint routes using hexagon-based networks. We describe the details of the algorithm and compare it with other schemes. Simulation results show that the proposed scheme achieves better performance in terms of message delivery ratio and network life time.
\end{abstract}

Index Terms-Multi-path routes, routing algorithms, wireless sensor networks.

\section{INTRODUCTION}

Wireless sensor network (WSN) is gaining popularities in critical applications. Composed of tens and thousands of sensor nodes, sensor network can work in the environment to which human cannot easily approach. Sensor networks often contain one or more sinks that provide centralized control. A sink typically serves as the access point for the user or as a gateway to another network. Large sensor networks can be composed of thousands of sensor nodes deployed in the field to observe a region. One of the advantages of wireless sensors networks is their ability to operate unattended in harsh environments. Given the remote area to be scrutinized, the short lifetime of the battery-powered sensors and the possibility of having damaged nodes during deployment, a large number of sensors are employed in most applications. It is envisioned that hundreds or even thousands of sensor nodes will be involved. Sensor networks have several major constraints: limited processing capability, limited storage capacity, limited bandwidth, and limited energy resource. Researchers are working to solve many of the limitations affecting sensor nodes and networks. Some research issues include improved node design, improved protocols associated with a sensor network and still others are working to resolve security issues [1].

Establishing single-path routing between the source and destination nodes is more common study topics in WSNs. However, compromise of nodes along the path would lead to failure of the path and loss of data. Furthermore, if routing path is compromised then the entire WSN is endangered. In sensitive applications, establishing reliability and availability

Manuscript received November 7, 2014; revised March 6, 2015. This work was supported by the Kumoh National Institute of Technology, Korea

Si Gwan Kim is with Kumoh National Institute of Technology, Korea (e-mail:sgkim999@gmail.com). is considered vital for an application to serve its objectives successfully. To offer multiple paths in order to enhance the availability, resilience and reliability of the network, many studies suggest various mechanisms. However, the use of multiple paths introduces additional security problems, since it makes data available at more locations, giving more opportunities to adversaries to compromise the data. Therefore, in sensitive environments it is important to protect the network from malicious actions in order to enhance and maintain the availability and reliability of the network.

The rest of the paper is organized as follows. In Section II, we introduce the related works. In Section III, we describe multi-path routing algorithm. In Section IV, we present the results of our simulations. Finally, we summarize our results in Section V.

\section{RELATED WORKS}

Sensor network is composed of many sensor nodes. As many sensor nodes are placed at the location where users want to get information, information is collected and the collected information is transmitted to node called sink. This sink is a node which can communicate with sensors in the sensor network. Sink transmits the information detected by sensor to users. Users may directly request sink to provide the data they want. Then, sink transmits includes user requirements in a kind of question to send them to sensors. The information collected in sensor is transmitted to sink through multi-hop sensor networks. The design of sensor network is affected by factors such as possibility of failure, expansibility, production cost, topology, restriction of hardware, transmitting media, power consumption, etc [2]-[4].

Routing in wireless sensor network is intensively studied these days. The existing routing protocol can be largely divided into three parts. Directed Diffusion, SPIN and Rumor Routing protocol are included in the data oriented routing technique, which sets route from node at specific location to sink node by sending queries when sink node needs specific area or specific attributes. Location based routing includes GEAR, LAR and GPSR protocol, which remove unnecessary routing set up by asking question to specific region by using location information. Cluster based routing protocol is to prevent unnecessary energy waste caused by redundant transmission of similar data of adjacent node and to reduce load on the relay node.

LEACH protocol and modified protocols [5]-[8] are the typical examples of cluster-based protocol. The operation of LEACH protocol is composed of 2 stages called round and it is formed of the repetition of such round. As adjacent sensor nodes usually have similar data, cluster head collects data 
from cluster member node to reduce energy waste caused by redundant transmission of information. Then, they are combined to be directly transmitted to sink node. Network life time is elongated by collecting data to evenly disperse energy consumption of all sensor nodes in the network and integrating them in sink node to equally replace cluster head with large energy in each round time unit.

PEGASIS is near optimal for data gathering application in sensor networks [9]. The key idea in PEGASIS is to form a chain among the sensor nodes so that each node will receive from and transmit to closer neighbor. Data gets aggregated while moving from node to node, and finally a designated node transmits aggregated data to the BS. Nodes take turns transmitting to the BS so that the average energy spent by each node per round is minimized. Building a chain to minimize the total length is similar to the traveling salesman problem. PEGASIS performs better than LEACH by eliminating the overhead of dynamic cluster formation, minimizing the distance non leader-nodes must transmit, limiting the number of transmissions and receives among all nodes, and using only one transmission to the BS per round.

Hybrid protocols like Adaptive Periodic TEEN(APTEEN) incorporate both proactive and reactive concepts . APTEEN first compute all routes and then improve the routes at the time of routing. But, this requires additional complexity to implement the threshold function and count time features [10], [11].

Directed Diffusion protocols are application specific and can save energy by selecting optimal paths by caching and processing data in the network. But, for data aggregation it needs time synchronization technique that is not very easy to achieve in WSNs. In addition, the overhead involved in recording information thus increasing the cost of a sensor node.

\section{OUR ALGORITHMS}

In this section, we propose our algorithms based on multi-path routes. In our system, we use hexagon-based coordinates as well as grid-based ones. A hexagon-based coordinate system has more advantages over a grid-based one in wireless sensor networks. First, when a sensor node transmits data over wireless links, its signal range would form a circle that is centered around its deployment location with the radius being the distance of signal propagation. And, a hexagon can be used to describe equal distance between two neighboring sensor nodes. In a grid-based coordinate system, the distance between two neighboring sensor nodes differs. When the neighboring node is located directly adjacent or diagonally in the grid-based system, its distance is one unit and square root of two units, respectively.

In our systems, nodes are place in the grid form $n \times n$ as in Fig. 1. We assume that transmission range is two hops for each node. Label of each node is numbered using two dimensional matrix.

$$
\{N(i, j) \mid i=0,1,2, \ldots, n-1, j=0,1,2, \ldots, n-1\}
$$

where, $n$ is the size of the network.
Four independent networks, $G_{0}, G_{1}, G_{2}$ and $G_{3}$, can be organized using the hexagon-based scheme as follows.

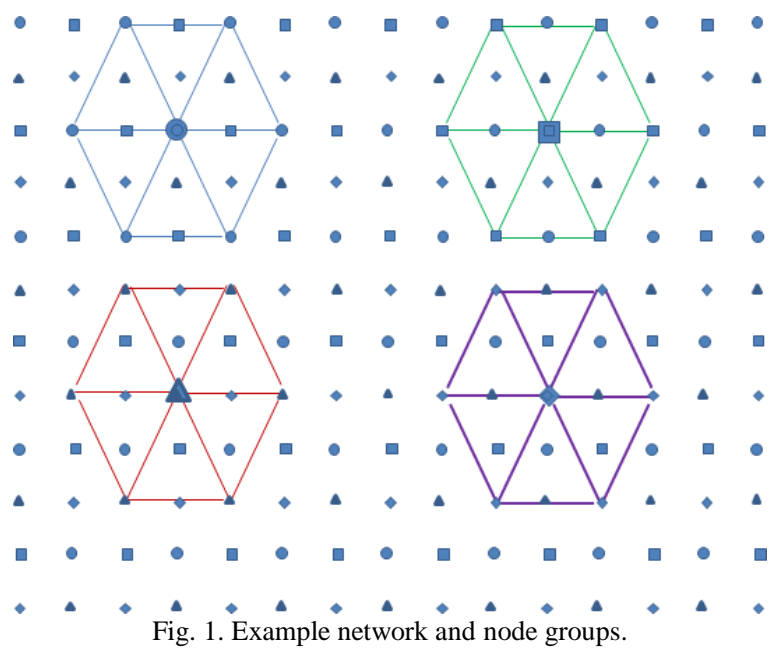

- $G_{0}=\{N(i, j) \mid i=0,2,4, \ldots, 2 m-2, j=0,2,4, \ldots, 2 m-2\}$

- $G_{1}=\{N(i, j) \mid i=0,2,4, \ldots, 2 m-2, j=1,3,7, \ldots, 2 m-1\}$

- $G_{2}=\{N(i, j) \mid i=1,3,7, \ldots, 2 m-1, j=0,2,4, \ldots, 2 m-2\}$

- $G_{3}=\{N(i, j) \mid i=1,3,7, \ldots, 2 m-1, j=1,3,5, \ldots, 2 m-1\}$

A node can be identified as a member of $G_{0}, G_{1}, G_{2}$ and $G_{3}$ according to the following rules.

- $i$ and $j$ value of node $N(i, j)$ are all even numbers: A member node of $G_{0}$

- $i$ and $j$ value of node $N(i, j)$ are even and odd number, respectively: A member node of $G_{1}$

- $i$ and $j$ value of node $N(i, j)$ are odd and even number, respectively: A member node of $G_{2}$

- $i$ and $j$ value of node $N(i, j))$ are all odd numbers: A member node of $G_{3}$

In Fig. 1, example source nodes of four networks are shown. An example member node in $G_{0}, G_{1}, G_{2}$ and $G_{3}$ is drawn as circle, rectangle, triangle and diamond, respectively. In this figure, an example of hexagon-based routing path is shown for each $G_{0}, G_{1}, G_{2}$ and $G_{3}$, with each source node drawn as big circle, big rectangle, big triangle and big diamond, respectively.

Our scheme has two kinds of hops, one-hop delivery and two-hop delivery. One-hop delivery is used for the distribution of message segments to the neighbor nodes, or last hop of the segments to the destination node. This one-hop is routed using grid-based coordinates. Hexagon-based two-hop delivery is used for the major hop for the routing.

When a node wants to send some messages to the destination node, a given message is divided into four segments, i.e. $w_{0}, w_{1}, w_{2}$ and $w_{3}$. Each segment except $w_{0}$ is delivered to three neighboring nodes. This delivery takes just one hop for each segment. Then each segment including $w_{0}$ is routed to the destination node. This uses hexagon-based routing. Our routing algorithms are as follows.

Step 1. A given message is divided into four segments, i.e. $w_{0}, w_{1}, w_{2}$ and $w_{3}$.

Step 2. Each segment $w_{0}, w_{1}, w_{2}$ and $w_{3}$ is delivered via $G_{0}$, $G_{1}, G_{2}$ and $G_{3}$, respectively.

Step 3. If source node is identified as $w_{0}, w_{1}, w_{2}$ or $w_{3}$, corresponding $G_{i}(i=0,1,2,3)$ will be used for its segment routing. Other three segments are delivered to the 
corresponding one-hop away neighboring nodes.

Step 4. Then all segments are arrived at the intermediate starting node, where each segment uses two-hop routing based on hexagon formation for further routing.

Step 5. When each segment is arrived at the intermediate starting node, each segment is routed to the destination node according to the pre-determined $G_{i}$.

Step 6. As each segment gets closer to the destination node, the last hop may be one-hop or two-hop routing for the destination node depending on the position of that node.

Fig. 2 shows an example of our routing. Source node ' $S$ ' has some messages for the destination node ' $D$ '. Node $S$ divides a message into four segments. First, only three segments are forwarded to the neighbor nodes using one-hop routing. This is shown in Fig. 2a). Now four segments including one segment generated for its own source node are ready to be routed to the destination node using two-hop routing. The final hop to the destination node $D$ uses one-hop delivery. This is shown in Fig. 2b).
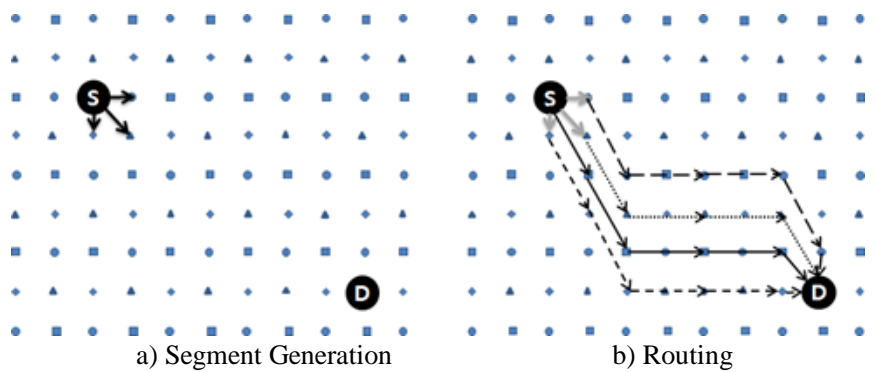

Fig. 2. Example routing.

\section{Simulations}

The performance of suggested algorithm is simulated and analyzed in this section. NS-2 is used to perform simulation to compare and analyze performance with the previous works, LEACH [5].

The size of network is $100 \mathrm{~m} \times 100 \mathrm{~m}$ and sink is located outside of network. Simulation environments are as follows: simulation time is $900 \mathrm{sec}$, packet size is 50 bytes, communication range is $15 \mathrm{~m}$, initial energy is $2 \mathrm{~J}$, aggregation energy is $5 \mathrm{~nJ}$, transmitter energy is $600 \mathrm{~mW}$, receiver energy is $300 \mathrm{~mW}$ and idle energy is $120 \mathrm{~mW}$. The performance of algorithm is observed with various parameters by increasing the number of message generating nodes from 20 to 60 . We have performed three simulations to evaluate our protocols as follows:

- Network Life Time: the number of alive nodes as time elapses

- Packet Delivery Ratio: percentage of packets sent by the source which reaches the sink depending on the number of source nodes

\section{A. Network Life Time}

Network lifetime of WSN is very important due to the limited energy. Here, three metrics are given to show the state of network energy consumption: the number of alive nodes as times elapse, the time the first node death because of energy shortage and the time of network death for different number of nodes.
Fig. 3 shows the results of the number of alive nodes. The proposed scheme performs better than the previous schemes. After 600 seconds, most of nodes die for both schemes. Fig. 4 and Fig. 5 show the results of the first node death time and last node death time, respectively. As the initial energy is bigger, the proposed scheme performs better than the previous schemes.

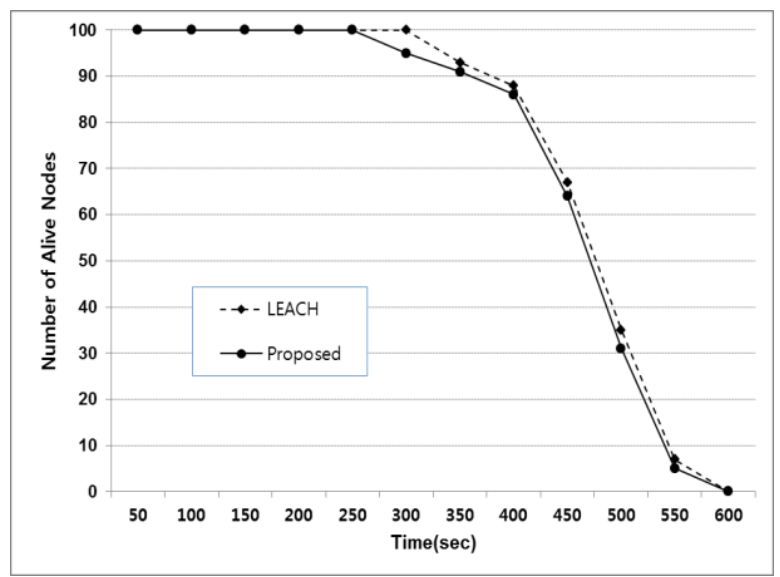

Fig. 3. Number of alive nodes.

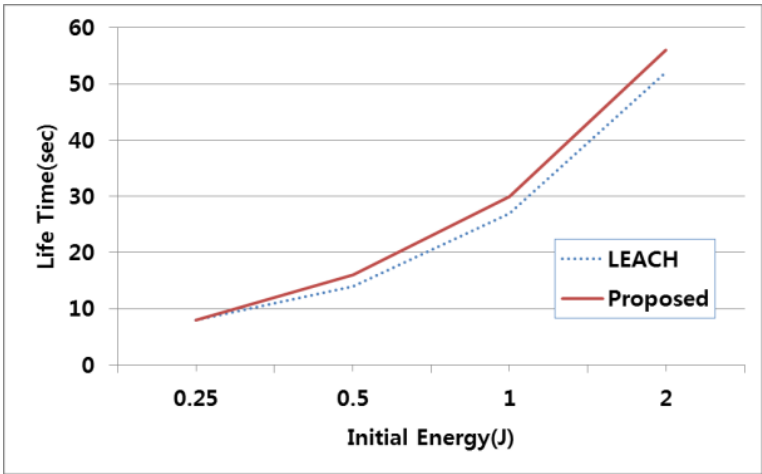

Fig. 4. Time to first node death.

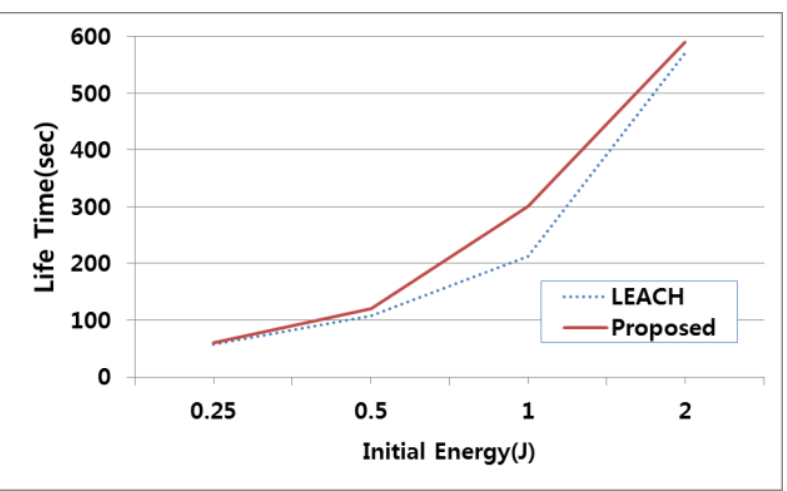

Fig. 5. Time to last node death

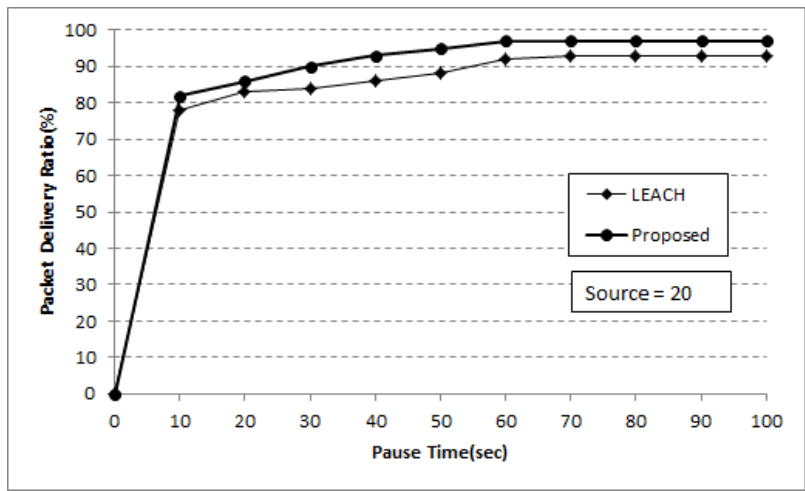

Fig. 6. Message delivery ratio (sources $=20$ ). 


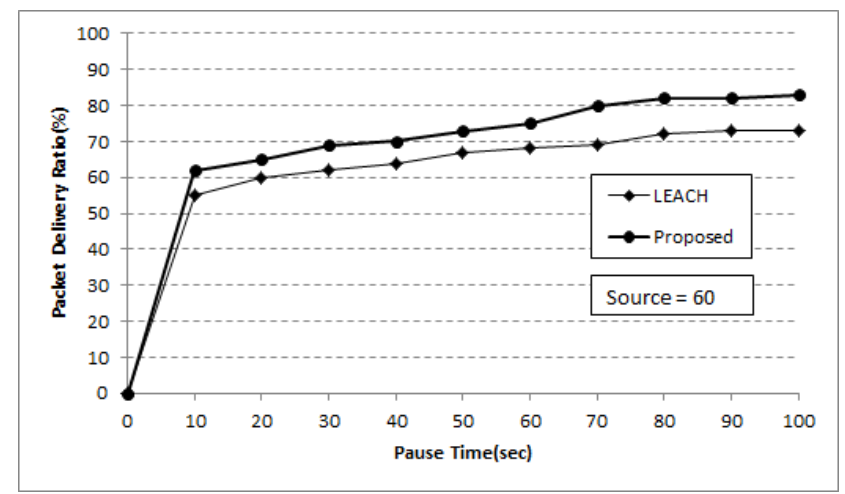

Fig. 7. Message delivery ratio (sources $=40)$.

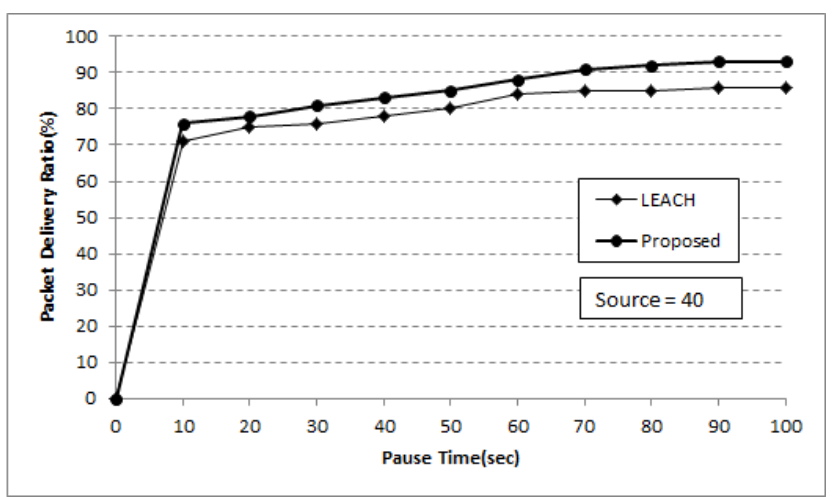

Fig. 8. Message delivery ratio (

\section{B. Message Delivery Ratio}

Fig. 6, Fig. 7 and Fig. 8 show message delivery ratio when the number of node was 100 , the interval time between messages changes from 0 second to 100 seconds and the number of message for each node is 20,40 and 60 . The suggested algorithm was found to have the packet delivery ratio higher than that of LEACH algorithm by about $5 \%$. Though our scheme generates more packets than the previous one, more routes are available for its routing, resulting in more successful message arrivals.

\section{CONCLUSIONS}

Sensor network is limited by battery capacity of sensor node that composes network, computation ability and the memory capacity. This paper suggests routing algorithm based on multi-path routes in sensor network. Simulation was performed in terms of network survival time and message delivery ratio to compare the performance of suggested algorithm with that of the previous method. Due to the more availabilities of routing paths, the suggested algorithm shows higher message delivery ratio than that of the existing method by some $5 \%$. In the future research, we consider secure routing algorithms based on key pre-distribution techniques. Our segmented messages scheme based on multi-path routes would be more suited to secure routing [12].

\section{REFERENCES}

[1] I. Akyildiz, S. Weilian, Y. Sankarasubramaniam, and E. Cayirci, "A survey on sensor networks," IEEE Communications Magazine, vol. 40, no. 8, pp. 102-114, 2002.

[2] I. F. Akyildiz, W. Su, Y. Sankarasubramaniam, and E. Cayirci, "A survey on sensor networks," IEEE Communications Magazine, vol. 40, pp. 102-114, August, 2002.

[3] D. D. Vergados et al., "Energy-efficient routing protocols in wireless sensor networks: A survey," Communications Surveys \& Tutorials, vol. 15, no. 2, 2013, pp. 551-591.

[4] J. N. Al-Karaki and A. E. Kamal, "Routing techniques in wireless sensor networks: A survey," Wireless Communications, vol. 11, pp 6-28, Dec., 2004.

[5] W. Heinzelman, J. Kulik, and H. Balakrishnan, "Adaptive Protocols for Information Dissemination in Wireless Sensor Networks," in Proc. 5th ACM/IEEE Mobicom Conference (MobiCom '99), Seattle, WA, August, 1999, pp. 174-185.

[6] W. Heinzelman, A. Chandrakasan, and H. Balakrishnan, "Energy-efficient communication protocol for wireless micro-sensor networks," in Proc. the 33rd Hawaii International Conference on System Sciences (HICSS '00), vol. 2, January, 2000, p. 10.

[7] W. R. Heinzelman, A. Chandrakasan, and H. Balakrishnan, "An application-specific protocol architecture for wireless microsensor networks," IEEE Transactions on Wireless Communications, vol. 1, no. 4 , pp. 660-670, Oct. 2002.

[8] S. Mohammad et al., "LEACH enhancements for wireless sensor networks based on energy model," in Proc. 2014 11th International Multi-Conference on Systems, Signals \& Devices (SSD), IEEE, 2014.

[9] L., Stephanie and C. S. Raghavendra, "PEGASIS: Power-efficient gathering in sensor information systems," Aerospace Conference Proceedings, vol. 3. IEEE, 2002.

[10] M. Arati and D. P. Agrawal, "APTEEN: A hybrid protocol for efficient routing and comprehensive information retrieval in wireless sensor networks," in Proc. Parallel and Distributed Processing Symposium, vol. 2. IEEE Computer Society, 2002.

[11] M. Arati and D. P. Agrawal, "TEEN: A routing protocol for enhanced efficiency in wireless sensor networks," in Proc. Parallel and Distributed Processing Symposium, vol. 3, IEEE Computer Society, 2001.

[12] L. Shuang et al., "Efficient multi-path protocol for wireless sensor networks," International Journal of Wireless and Mobile Networks, vol. 2, no. 1, 2010, pp. 110-130.

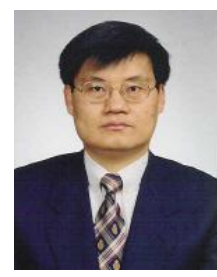

Si-Gwan Kim received the B.S. degree in computer science from Kyungpook Nat'l University in 1982 and the M.S. and Ph.D. degrees in computer science from KAIST, Korea, in 1984 and 2000, respectively. He worked for Samsung Electronics until 1988 and then he joined the Department of Computer Software Engineering, Kumoh National Institute of Technology, Gumi, Korea, as a professor. His research interests include sensor networks, mobile programming and parallel processing. 